\title{
COORDENAÇÃO DO NADO BORBOLETA: ESTUDO PILOTO SOBRE OS EFEITOS DA VELOCIDADE DE NADO E DAS AÇÕES INSPIRATÓRIAS
}

\author{
MS. RICARDO PETERSON SILVEIRA \\ Mestre em Ciências do Movimento Humano pela Escola de Educação Física da \\ Universidade Federal do Rio Grande do Sul (ESEF/UFRGS) \\ Laboratório de Pesquisa do Exercício da ESEF/UFRGS \\ (Porto Alegre - Rio Grande do Sul - Brasil) \\ E-mail: ricardopetersons@yahoo.com.br \\ MS. FELIPE COLLARES MORÉ \\ Mestre em Ciências do Movimento Humano pela Escola de Educação Física da \\ Universidade Federal do Rio Grande do Sul (ESEF/UFRGS) \\ Laboratório de Pesquisa do Exercício da ESEF/UFRGS \\ (Porto Alegre - Rio Grande do Sul - Brasil) \\ E-mail:fcmore@gmail.com
}

\author{
DR. FLÁVIO ANTÔNIO DE SOUZA CASTRO \\ Professor da Escola de Educação Física da Universidade Federal do Rio Grande do Sul (ESEF/UFRGS), \\ Doutorado em Ciências do Movimento Humano pela ESEF/UFRGS \\ Laboratório de Pesquisa do Exercício da ESEF/UFRGS \\ (Porto Alegre - Rio Grande do Sul - Brasil) \\ E-mail: souza.castro@ufrgs.br
}

\begin{abstract}
DR. CARLOS BOLLI MOTA
Professor do Centro de Educação Física e Esporte da Universidade Federal de Santa Maria (CEFD/

UFSM), Doutorado em Ciência do Movimento Humano pelo CEFD/UFSM

Laboratório de Ensino e Pesquisa do Movimento Humano do CEFD/UFSM

(Santa Maria - Rio Grande do Sul - Brasil)

E-mail: bollimota@gmail.com
\end{abstract}

\begin{abstract}
RESUMO
A proposta deste estudo piloto foi verificar os efeitos da velocidade de nado e dos ciclos inspiratórios sobre os parâmetros coordenativos do nado borboleta. Participaram quatro nadadores de nível regional, com desempenho médio de $69 \pm 3,6 \%$ do recorde mundial na prova de $100 \mathrm{~m}$ borboleta em piscina de $25 \mathrm{~m}$. Em resposta ao incremento da velocidade de nado, entre os principais resultados, destaca-se: redução da duração absoluta dos ciclos (de 2,01 $\pm 0,43 \mathrm{spara} 1,07 \pm 0,09 \mathrm{~s}$, entre velocidade relativa aos $200 \mathrm{~m}$ e velocidade máxima em $25 \mathrm{~m}$, com inspiração frontal e similar comportamento para ciclos não inspiratórios); marcado aumento na duração das fases propulsivas da braçada, expressas em \% da duração média de quatro ciclos de braçada (fase B de 14,6 $\pm 3,2 \%$ para 22,8 \pm 4,7\% e fase $C$ de 12,9
\end{abstract}


$\pm 1,0$ para 19,1 $\pm 0,9 \%$ com inspiração frontal e similar comportamento para ciclos não inspiratórios), bem como maior sincronismo entre as ações da braçada e da pernada.

PALAVRAS-CHAVE: Natação; análise de vídeo; nado borboleta; coordenação.

\section{INTRODUÇÃO}

nado borboleta é considerado uma técnica simultânea, quanto à ação dos membros superiores e membros inferiores, e uma técnica descontínua em relação às ações propulsivas de braços e pernas (BARBOSA, 2004). Esta descontinuidade nas ações propulsivas tem sido estudada por meio da análise da coordenação, nas diferentes técnicas de nado, principalmente a partir do ano 2000, com o grupo da Universidade de Ruão (França) (CHOLLET et al., 2000; CHOLLET et al., 2004; CHOLLET et al., 2006; CHOLLET et al., 2008). O conceito de coordenação em natação, a partir da organização temporal das fases propulsivas de braçada e pernada, como um índice de coordenação, foi inicialmente proposto para o nado crawl (CHOLLET; CHALIES; CHATARD, 2000), técnica de análise na qual é verificada a coordenação entre braçadas que são executadas com os dois braços alternadamente, ao contrário do nado borboleta. Este parâmetro de coordenação é verificado por meio do intervalo de tempo entre o final da ação propulsiva de um braço e o início da ação propulsiva do outro, podendo-se classificar os modelos de coordenação, nos nados alternados, em captura, oposição ou sobreposição (SCHNITZLER et al., 2008).

nado crawl é o único dos estilos competitivos possível de ser executado, teoricamente, sob continuidade constante entre as ações propulsivas. As demais técnicas de nado apresentam limitações biomecânicas, seja pela mobilidade articular, no nado costas, seja pela simultaneidade nas ações dos membros, nos nados peito e borboleta (SEIFERT; CHOLLET, 2009). Chollet et al. (2006) verificaram o grau de sincronismo entre os pontos-chave da braçada e da pernada na técnica de borboleta em nadadores de elite, propondo quatro tempos relativos para a mensuração da coordenação. A coordenação braçada-pernada mostrou adaptações com relação ao incremento da velocidade de nado (VN) e do nível de experiência, havendo um maior sincronismo entre as ações propulsivas tanto nas velocidades mais elevadas, quanto em atletas de nível mais elevado (SEIFERT et al., 2007; SEIFERT et al., 2008).

Um dos aspectos de maior relevância para o desempenho na técnica de borboleta diz respeito à execução e à frequência de ciclos inspiratórios (ALVES; CUNHA; GOMES-PEREIRA, 1999; BARBOSA et al., 1999). O ciclo de inspiração frontal consiste na execução de uma extensão cervical, com a qual o rosto do nadador rompe a superfície da água. Tal movimento é essencial para o desempenho nesta técnica, devido à necessidade fisiológica de ventilação ao longo de provas com distâncias que variam de 50 m a 200 m, e ao longo das sessões de treinamento. Du- 
rante os ciclos não inspiratórios, o nadador executa uma flexão cervical, mantendo a face voltada para a água, podendo romper ou não a sua superfície (BARBOSA, 2002; 2004). Ainda, um ciclo completo do nado borboleta é composto por uma braçada e duas pernadas, assim, de modo teórico (nem todos os nadadores executam os movimentos nos tempos descritos a seguir), quando os braços entram na água (início do ciclo), ocorre uma primeira fase descendente da pernada, enquanto o nadador executa a fase submersa da braçada, ocorre a primeira fase ascendente da pernada, quando há finalização da braçada, imediatamente antes das mãos saírem da água, ocorre a segunda fase descendente da pernada e, por fim, enquanto os braços são recuperados, ocorre a segunda fase ascendente da pernada (BARBOSA, 2002; 2004).

Os ciclos de inspiração frontal podem levar a uma maior amplitude de deslocamento vertical da cabeça, maior área frontal projetada e, consequentemente, a maior arrasto hidrodinâmico (BARBOSA et al., I999; BARBOSA, 2002; 2004). No entanto, não está clara a repercussão da execução de ações inspiratórias sobre a coordenação de nado. Desta forma, se torna relevante a análise da técnica do nado borboleta, considerando ( I ) a importância da compreensão das possíveis respostas da coordenação de nado às diferentes velocidades de nado e ações inspiratórias, (2) a escassez de estudos na área e (3) o método recentemente descrito por Chollet et al. (2006) para determinação dessas respostas.

Assim, o objetivo deste estudo piloto consiste em descrever a coordenação e duração das fases da braçada e da pernada no nado borboleta, em diferentes velocidades de nado, sob condição de ciclos de inspiração frontal e ciclos não-inspiratórios, de acordo com o descrito por Chollet et al. (2006).

\section{MATERIAIS E MÉTODOS}

\section{PARTICIPANTES}

Participaram deste estudo quatro nadadores de nível regional (idade média: 19,7 \pm ।,3 anos; estatura média: 178,9 \pm 4,5 cm; envergadura média: । $85 \pm 8,2$ cm; massa corporal média: 72,4 \pm 4,9 kg; desempenho médio nos 100 m borboleta: $69 \pm 3,6 \%$ do recorde mundial em piscina de $25 \mathrm{~m}$ à época de realização das coletas, o que indica um desempenho médio, na prova, de aproximadamente 63 s), especialistas nas provas de borboleta (50, 100 ou 200 m). $\bigcirc$ estudo obedeceu aos critérios de ética em pesquisas com humanos, resolução n 196, de 10 de outubro de 1996 (CNS). Todos os participantes foram previamente informados dos objetivos, procedimentos e riscos envolvidos nesta pesquisa. Após avaliação por Comitê de Ética em Pesquisa em Seres Humanos, este projeto de pesquisa foi aprovado sob número 19719. 
Em uma piscina de $25 \mathrm{~m}$ coberta e aquecida, os nadadores executaram, individualmente, oito repetições de $25 \mathrm{~m}$, partindo de dentro da piscina, em velocidades relativas à velocidade média nas provas de 200 m (V200), 100 m (VI 00), 50 m (V50) e máxima velocidade de nado relativa à distância de 25 m (Vmáx) para as condições de execução de ciclos de inspiração frontal e de ciclos não-inspiratórios, em uma única sessão. As repetições foram realizadas em ordem aleatória, tanto para o fator velocidade de nado, quanto para o fator modelo inspiratório. Para evitar possíveis efeitos da fadiga sobre a técnica de nado, foi observado um intervalo de, no mínimo, três minutos em repouso passivo entre cada repetição. Como eram nadadores competitivos, acostumados a séries mais intensas e com menor intervalo, pode-se afirmar que não houve efeitos relacionados à fadiga sobre a técnica de nado, até mesmo porque foram apenas duas repetições máximas de $25 \mathrm{~m}$.

Os nadadores foram orientados a respirar em todas as braçadas, com exceção da primeira, nas repetições em ciclos de inspiração frontal. Da mesma forma, os nadadores foram orientados a não respirar nas repetições em ciclos de braçadas não inspiratórios. Os tempos-alvo para cada repetição foram baseados nos melhores desempenhos dos sujeitos na temporada (nas três provas oficiais de borboleta), sendo aceita uma margem de erro de $\pm 2,5 \%$ sobre o mesmo. Quando necessário, ou seja, quando o desempenho era diferente em mais de 2,5\%, para cima ou para baixo do tempo-alvo, o nadador realizava novamente a repetição específica.

\section{COLETA E ANÁLISE DOS VÍDEOS}

Foi realizada videogrametria subaquática utilizando-se uma câmera (JVC, GR-DVL9800), operando a uma frequência de amostragem de $25 \mathrm{~Hz}$, a qual estava alocada em uma caixa estaque imersa a 0,5 m de profundidade, e a uma distância de oito metros da raia utilizada para os testes. $\bigcirc$ deslocamento da câmera foi realizado manualmente por meio de um carrinho sobre trilhos posicionados na lateral da piscina. A distância da câmera ao nadador possibilitou a coleta de imagens do corpo inteiro do nadador, com visualização da entrada da mão na água e movimentos dos pés.

As fases da braçada e da pernada foram determinadas por meio de uma análise quadro a quadro realizada de maneira independente por três avaliadores experientes, utilizando o software VirtualDub I.8.7, sendo que todas as análises, de quatro ciclos completos, foram realizadas a partir do segundo ciclo de braçadas após a saída, para reduzir os efeitos do impulso sobre a parede da piscina. Os seguintes critérios foram obedecidos: ( I ) quando o ponto chave determinado foi igual para, no mínimo, dois dos avaliadores, este foi considerado como correto; (2) se os pontos 
chave determinados tiveram uma diferença de até $0,08 \mathrm{~s}$ (dois quadros) para mais e/ou para menos, para os três avaliadores, foi considerado o valor intermediário; e (3) no caso de divergências superiores a 0,08 s (dois quadros) entre os pontos determinados pelos três avaliadores, foi realizada uma reunião entre, no mínimo, dois deles quando, após nova análise dos vídeos, foi determinado o ponto chave válido.

\section{FASES DA BRAÇADA E DA PERNADA}

As fases da braçada e da pernada foram determinadas segundo Chollet et al. (2006). A braçada foi dividida em quatro fases, representando a média de quatro braçadas analisadas:

- Entrada e apoio (A): tempo decorrido entre o ponto de entrada das mãos na água até o ponto imediatamente anterior ao movimento das mãos para trás. Puxada (B): tempo decorrido entre o ponto imediatamente anterior ao movimento das mãos para trás até o ponto em que as mãos estejam posicionadas verticalmente em relação ao ombro. - Empurrada (C): tempo decorrido entre o ponto em que as mãos estejam posicionadas verticalmente em relação aos ombros até o ponto de saída das mãos da água. - Recuperação (D): tempo decorrido entre o ponto de saída até o ponto de entrada das mãos novamente na água.

As fases $B$ e $C$ são consideradas fases propulsivas, ao passo que as fases $A$ e $D$ são consideradas fases não-propulsivas da braçada. Os ciclos de pernadas foram divididos em quatro fases, também representando o valor médio de quatro ciclos completos:

- Fase descendente I (DI): tempo decorrido entre o ponto de mudança de direção superior e o ponto de mudança de direção inferior da primeira pernada. - Fase ascendente I (A I): tempo decorrido entre o ponto de mudança de direção inferior e o ponto de mudança de direção superior da primeira pernada. - Fase descendente 2 (D2): tempo decorrido entre o ponto de mudança de direção superior e o ponto de mudança de direção inferior da segunda pernada. - Fase ascendente 2 (A2): tempo decorrido entre o ponto de mudança de direção inferior e o ponto de mudança de direção superior da segunda pernada DI e D2 são consideradas fases propulsivas da pernada, ao passo que Al e A2 são consideradas fases não-propulsivas da pernada. As durações das fases, da braçada e da pernada, foram mensuradas com uma precisão de 0,04 segundos, sendo expressa em valores percentuais da duração total média de quatro ciclos completos.

\section{COORDENAÇÃO BRAÇADA-PERNADA}

A coordenação braçada-pernada foi mensurada por meio de quatro tempos relativos entre pontos-chave da braçada e da pernada (TI, T2, T3 e T4), e de um índice global da coordenação de nado (TTG), conforme proposto por Chollet et 
al. (2006). A obtenção desses tempos relativos se dá pela diferença de tempo entre um ponto-chave da pernada e o seu respectivo ponto-chave da braçada, como descrito a seguir: - TI: tempo entre o ponto de entrada das mãos na água e o ponto de mudança de direção superior da primeira pernada. - T2: tempo entre o ponto imediatamente anterior ao movimento das mãos para trás e o ponto de mudança de direção inferior da primeira pernada. - T3: tempo entre o ponto em que as mãos estejam posicionadas verticalmente em relação aos ombros e o ponto de mudança de direção superior da segunda pernada. - T4: tempo entre o ponto de saída das mãos da água e o ponto de mudança de direção inferior da segunda pernada. - TTG: diferença de tempo total entre os pontos-chave da braçada e da pernada. Somatório, em segundos, dos tempos absolutos de TI, T2, T3 e T4, expresso em percentual da duração total de um ciclo completo de pernadas.

A Figura I ilustra como são obtidos os tempos relativos para mensuração da coordenação, considerando pontos-chave específicos da braçada e da pernada. Cada ponto-chave é ligado por uma linha contínua, cujos vales representam as fases propulsivas de braços e pernas.
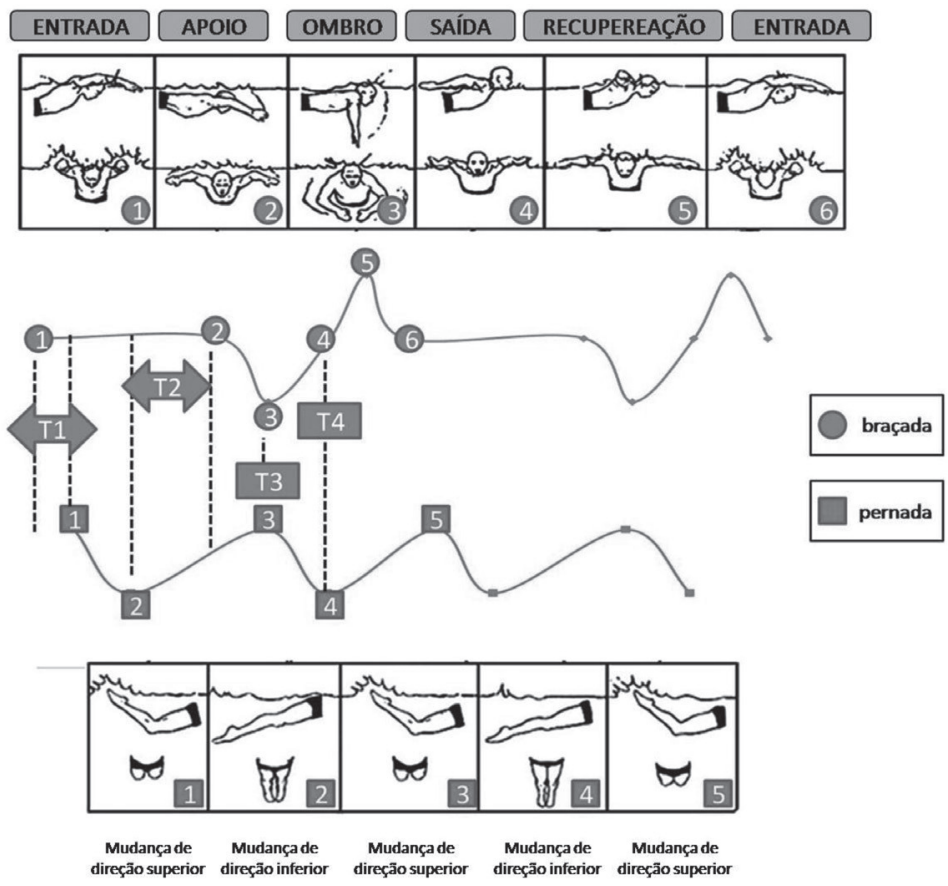

Figura I: Identificação dos pontos-chave para determinação da coordenação entre membros na técnica de borboleta.

Fonte: Adaptado de CHOLLET et al. (2006). 
Devido ao tamanho da amostra, de acordo com um estudo piloto, para a análise estatística optou-se por procedimentos descritivos, com o cálculo das médias e desvios-padrão das variáveis analisadas.

\section{RESULTADOS}

As velocidades de nado, em média, para V200, VI00, V50 e Vmáx foram de, respectivamente, I,45 $\pm 0,08 \mathrm{~m} \cdot \mathrm{s}^{-1} ; \mathrm{I}, 63 \pm 0,09 \mathrm{~m} \cdot \mathrm{s}^{-1} ; \mathrm{I}, 78 \pm 0,05 \mathrm{~m} \cdot \mathrm{s}^{-1} \mathrm{e}$ $1,88 \pm 0,07 \mathrm{~m} \cdot \mathrm{s}^{-1}$. Esses valores de velocidade de nado correspondem às médias das repetições com e sem respiração, calculadas conjuntamente.

\section{DURAÇÃO DAS FASES DA BRAÇADA E DA PERNADA}

Em termos absolutos, a Tabela I apresenta as durações médias dos ciclos de braçadas e de pernadas, para as quatro velocidades de nado e dois modelos respiratórios.

Tabela I - Duração média de quatro ciclos de braçada e de pernada, de acordo com a velocidade de nado e ciclos inspiratórios e não-inspiratórios (média \pm desvio-padrão), $n=4$

\begin{tabular}{ccccc}
\hline & \multicolumn{2}{c}{ Ciclos inspiratórios } & \multicolumn{2}{c|}{ Ciclos não-inspiratórios } \\
Duração ciclo & $\begin{array}{c}\text { Duração ciclo } \\
\text { braçadas (s) }\end{array}$ & $\begin{array}{c}\text { Duração ciclo } \\
\text { braçadas (s) }\end{array}$ & $\begin{array}{c}\text { Duração ciclo } \\
\text { pernadas (s) }\end{array}$ \\
\hline V200 & $2,01 \pm 0,43$ & $2,01 \pm 0,43$ & $1,95 \pm 0,34$ & $1,94 \pm 0,35$ \\
V100 & $1,51 \pm 0,15$ & $1,51 \pm 0,14$ & $1,44 \pm 0,09$ & $1,44 \pm 0,08$ \\
V50 & $1,29 \pm 0,12$ & $1,29 \pm 0,13$ & $1,18 \pm 0,12$ & $1,19 \pm 0,13$ \\
Vmáx & $1,07 \pm 0,09$ & $1,06 \pm 0,11$ & $1,05 \pm 0,11$ & $1,05 \pm 0,14$ \\
\hline
\end{tabular}

A Tabela 2 apresenta os resultados das durações relativas das fases da braçada em relação à duração total dos ciclos de braçada nas quatro velocidades de nado analisadas, para ciclos respiratórios e não-respiratórios. 
Tabela 2 - Duração das fases da braçada de acordo com a velocidade de nado (média \pm erro-padrão), em percentual, sob execução de ciclos de inspiratórios e não-inspiratórios, nas quatro velocidades de nado, em percentual da duração média de quatro ciclos. P: duração das fases propulsivas e NP: duração das fases não propulsivas, $\mathrm{n}=4$

\begin{tabular}{|c|c|c|c|c|c|c|c|c|}
\hline & \multicolumn{2}{|c|}{ V200 } & \multicolumn{2}{|c|}{ VI00 } & \multicolumn{2}{|c|}{ V50 } & \multicolumn{2}{|c|}{ Vmáx } \\
\hline & $\begin{array}{l}\text { Insp } \\
(\%)\end{array}$ & $\begin{array}{c}N \text {-insp } \\
(\%)\end{array}$ & $\begin{array}{l}\text { Insp } \\
(\%)\end{array}$ & $\begin{array}{c}N-\text { insp } \\
(\%)\end{array}$ & Insp (\%) & $\begin{array}{c}N \text { - insp } \\
(\%)\end{array}$ & $\operatorname{lnsp}(\%)$ & $N$ - insp (\%) \\
\hline Fase A & $\begin{array}{c}53,3 \\
\pm \\
19,4\end{array}$ & $\begin{array}{c}48,7 \\
\pm \\
17,3\end{array}$ & $\begin{array}{c}43,3 \\
\pm \\
7,5\end{array}$ & $\begin{array}{c}40,1 \pm \\
5,7\end{array}$ & $\begin{array}{c}37,2 \pm \\
6,3\end{array}$ & $\begin{array}{c}31,5 \pm \\
5,2\end{array}$ & $\begin{array}{c}28,9 \pm \\
5,9\end{array}$ & $\begin{array}{c}29,0 \pm \\
7,9\end{array}$ \\
\hline Fase B & $\begin{array}{c}14,6 \\
\pm \\
3,2\end{array}$ & $\begin{array}{c}15,5 \\
\pm \\
1,7\end{array}$ & $\begin{array}{c}18,5 \\
\pm \\
1,4\end{array}$ & $\begin{array}{c}16,6 \pm \\
1,8\end{array}$ & $\begin{array}{c}20,5 \pm \\
2,4\end{array}$ & $\begin{array}{c}20,7 \pm \\
3,2\end{array}$ & $\begin{array}{c}22,8 \pm \\
4,7\end{array}$ & $\begin{array}{c}22,6 \pm \\
4,4\end{array}$ \\
\hline Fase C & $\begin{array}{c}12,9 \\
\pm \\
1,0\end{array}$ & $\begin{array}{c}|5,| \\
\pm \\
\mid, 7\end{array}$ & $\begin{array}{c}15,7 \\
\pm \\
\mid, 5\end{array}$ & $\begin{array}{c}17,8 \pm \\
1,9\end{array}$ & $\begin{array}{c}17,0 \pm \\
1,0\end{array}$ & $\begin{array}{c}19,0 \pm \\
2,2\end{array}$ & $\begin{array}{c}19,1 \pm \\
0,9\end{array}$ & $\begin{array}{c}19,7 \pm \\
1,6\end{array}$ \\
\hline Fase D & $\begin{array}{c}18,9 \\
\pm \\
2,7\end{array}$ & $\begin{array}{c}20,5 \\
\pm \\
2,1\end{array}$ & $\begin{array}{c}22,0 \\
\pm \\
3,3\end{array}$ & $\begin{array}{c}25,3 \pm \\
1,8\end{array}$ & $\begin{array}{c}25,3 \pm \\
3,7\end{array}$ & $\begin{array}{c}28,6 \pm \\
3,5\end{array}$ & $\begin{array}{c}28,5 \pm \\
2,4\end{array}$ & $\begin{array}{c}28,0 \pm \\
2,5\end{array}$ \\
\hline $\begin{array}{l}P \\
(B+C)\end{array}$ & $\begin{array}{c}27,2 \\
\pm \\
2,6\end{array}$ & $\begin{array}{c}30,6 \\
\pm \\
3,1\end{array}$ & $\begin{array}{c}34,2 \\
\pm \\
1,2\end{array}$ & $\begin{array}{c}34,5 \pm \\
3,1\end{array}$ & $\begin{array}{c}37,5 \pm \\
1,8\end{array}$ & $\begin{array}{c}39,8 \pm \\
4,2\end{array}$ & $\begin{array}{c}42,0 \pm \\
5,1\end{array}$ & $\begin{array}{c}42,3 \pm \\
5,6\end{array}$ \\
\hline NP & $\begin{array}{c}72,6 \\
\pm \\
18,7\end{array}$ & $\begin{array}{c}69,2 \\
\pm \\
16,0\end{array}$ & $\begin{array}{c}65,3 \\
\pm \\
9,2\end{array}$ & $\begin{array}{c}65,4 \pm \\
5,9\end{array}$ & $\begin{array}{c}62,5 \pm \\
8,2\end{array}$ & $\begin{array}{c}60,1 \pm \\
7,9\end{array}$ & $\begin{array}{c}57,4 \pm \\
3,9\end{array}$ & $\begin{array}{c}57,1 \pm \\
8,7\end{array}$ \\
\hline
\end{tabular}

A Tabela 3 apresenta os resultados das durações relativas das fases das pernadas em relação à duração total dos ciclos nas quatro velocidades de nado analisadas, para ciclos respiratórios e não-respiratórios.

Tabela 3 - Duração das fases da pernada em média \pm desvio-padrão (duas fases descendentes e duas fases ascendentes), sob execução de ciclos inspiratórios e não-inspiratórios, nas quatro velocidades de nado, em percentual da duração média de quatro ciclos, $\mathrm{n}=4$

\begin{tabular}{|c|c|c|c|c|c|c|c|c|}
\hline & \multicolumn{2}{|c|}{ V200 } & \multicolumn{2}{|c|}{ VIOO } & \multicolumn{2}{|c|}{ V50 } & \multicolumn{2}{|c|}{ Vmáx } \\
\hline & $\operatorname{lnsp}(\%)$ & $\begin{array}{c}N \text {-insp } \\
(\%)\end{array}$ & $\begin{array}{l}\text { Insp } \\
(\%)\end{array}$ & $\begin{array}{c}N \text { - insp } \\
(\%)\end{array}$ & $\begin{array}{l}\text { Insp } \\
(\%)\end{array}$ & $\begin{array}{c}N \text { - insp } \\
(\%)\end{array}$ & $\begin{array}{l}\text { Insp } \\
(\%)\end{array}$ & $\mathrm{N}$ - insp (\%) \\
\hline $\begin{array}{c}\text { Descen } \\
\mid\end{array}$ & $\begin{array}{c}12,1 \pm \\
2,5\end{array}$ & $\begin{array}{c}13,5 \pm \\
1,9\end{array}$ & $\begin{array}{c}15,0 \pm \\
2,4\end{array}$ & $\begin{array}{c}13,5 \pm \\
1,6\end{array}$ & $\begin{array}{c}16,4 \pm \\
2,0\end{array}$ & $\begin{array}{c}17,6 \pm \\
3,8\end{array}$ & $\begin{array}{c}17,9 \pm \\
1,5\end{array}$ & $\begin{array}{c}17,3 \pm \\
2,3\end{array}$ \\
\hline
\end{tabular}




\begin{tabular}{ccccccccc} 
Ascen & $42,0 \pm$ & $41,8 \pm$ & $39,4 \pm$ & $40,4 \pm$ & $36,2 \pm$ & $35,5 \pm$ & $35,3 \pm$ & $36,9 \pm$ \\
I & 12,7 & 10,3 & 9,0 & 6,6 & 7,9 & 7,5 & 8,0 & 8,1 \\
Descen & $12,5 \pm$ & $13,5 \pm$ & $13,9 \pm$ & $14,2 \pm$ & $17,0 \pm$ & $17,0 \pm$ & $17,9 \pm$ & $17,3 \pm$ \\
2 & 2,5 & 2,9 & 2,8 & 1,6 & 2,2 & 3,8 & 3,7 & 0,9 \\
Ascen & $32,9 \pm$ & $30,7 \pm$ & $31,7 \pm$ & $31,7 \pm$ & $30,0 \pm$ & $29,6 \pm$ & $28,7 \pm$ & $28,0 \pm$ \\
2 & 9,4 & 6,7 & 4,7 & 2,9 & 3,4 & 5,4 & 6,9 & 5,9 \\
\hline
\end{tabular}

\section{COORDENAÇÃO BRAÇADA-PERNADA}

A Tabela 4 apresenta a coordenação entre braçada e pernada (TI a T4) e a coordenação geral do nado (TTG), em valores relativos à duração média de quatro ciclos.

Tabela 4 - Coordenação braçada-pernada (média \pm desvio-padrão), sob execução de ciclos de inspiratórios e não-inspiratórios, nas quatro velocidades de nado, em percentual da duração média de quatro ciclos, $n=4$

\begin{tabular}{|c|c|c|c|c|c|c|c|c|}
\hline & \multicolumn{2}{|c|}{ V200 } & \multicolumn{2}{|c|}{ V100 } & \multicolumn{2}{|c|}{ V50 } & \multicolumn{2}{|c|}{ Vmáx } \\
\hline & $\begin{array}{l}\text { Insp } \\
(\%)\end{array}$ & $\begin{array}{c}N \text { - insp } \\
(\%)\end{array}$ & $\begin{array}{l}\text { Insp } \\
(\%)\end{array}$ & $\begin{array}{c}\mathrm{N} \text { - insp } \\
(\%)\end{array}$ & $\begin{array}{l}\text { Insp } \\
(\%)\end{array}$ & $\begin{array}{c}N-\text { insp } \\
(\%)\end{array}$ & $\begin{array}{l}\text { Insp } \\
(\%)\end{array}$ & $\begin{array}{c}N \text { - insp } \\
(\%)\end{array}$ \\
\hline TI & $0,05 \pm 0,05$ & $\begin{array}{c}0,11 \\
\pm 0,05\end{array}$ & $\begin{array}{c}0,09 \\
\pm 0,04\end{array}$ & $\begin{array}{c}0,07 \pm \\
0,03\end{array}$ & $\begin{array}{c}0,05 \pm \\
0,03\end{array}$ & $\begin{array}{l}0,0 \pm \\
0,04\end{array}$ & $\begin{array}{c}-0,02 \pm \\
0,03\end{array}$ & $\begin{array}{c}-0,01 \pm \\
0,03\end{array}$ \\
\hline T2 & $-0,68 \pm 0,33$ & $\begin{array}{c}-0,58 \pm \\
0,28\end{array}$ & $\begin{array}{c}0,34 \pm \\
0,11\end{array}$ & $\begin{array}{c}-0,32 \pm \\
0,08\end{array}$ & $\begin{array}{c}-0,22 \pm \\
0,07\end{array}$ & $\begin{array}{c}-0,16 \pm \\
0,07\end{array}$ & $\begin{array}{c}-0,14 \pm \\
0,07\end{array}$ & $\begin{array}{c}-0,14 \pm \\
0,05\end{array}$ \\
\hline T3 & $-0,13 \pm 0,21$ & $\begin{array}{c}-0,07 \pm \\
0,17\end{array}$ & $\begin{array}{c}-0,02 \pm \\
0,03\end{array}$ & $\begin{array}{c}0,03 \pm \\
0,02\end{array}$ & $\begin{array}{c}-0,01 \pm \\
0,05\end{array}$ & $\begin{array}{c}0,02 \pm \\
0,01\end{array}$ & $\begin{array}{c}-0,01 \pm \\
0,03\end{array}$ & $\begin{array}{c}0,02 \pm \\
0,03\end{array}$ \\
\hline T4 & $-0,14 \pm 0,21$ & $\begin{array}{c}-0,10 \pm \\
0,12\end{array}$ & $\begin{array}{c}-0,05 \pm \\
0,08\end{array}$ & $\begin{array}{c}-0,02 \pm \\
0,05\end{array}$ & $\begin{array}{c}-0,01 \pm \\
0,04\end{array}$ & $\begin{array}{l}0,0 \pm \\
0,06\end{array}$ & $\begin{array}{c}-0,02 \pm \\
0,08\end{array}$ & $\begin{array}{c}-0,01 \pm \\
0,04\end{array}$ \\
\hline TTG & $1,10 \pm 0,73$ & $\begin{array}{c}0,9 \pm \\
0,53\end{array}$ & $\begin{array}{c}0,53 \pm \\
0,19\end{array}$ & $\begin{array}{c}0,45 \pm \\
0,13\end{array}$ & $\begin{array}{c}0,34 \pm \\
0,11\end{array}$ & $\begin{array}{c}0,27 \pm \\
0,09\end{array}$ & $\begin{array}{c}0,24 \pm \\
0,13\end{array}$ & $\begin{array}{c}0,22 \pm \\
0,06\end{array}$ \\
\hline
\end{tabular}

\section{DISCUSSÃO}

A discussão dos resultados obtidos é dividida em dois tópicos principais. No primeiro, são discutidos os efeitos da velocidade de nado, e, no segundo, os efeitos das ações inspiratórias sobre a duração das fases e a coordenação braçada-pernada.

\section{EFEITO DA VELOCIDADE DE NADO}

O protocolo aplicado, baseado em velocidades relativas aos melhores tempos de cada nadador nas provas de 200, 100 e 50 m nado borboleta e velocidade máxima em 25 m, foi eficiente para gerar diferentes velocidades de nado. A duração, 
em termos absolutos, das braçadas e pernadas (Tabela I) reduziu, ao passo que a velocidade de nado aumentou. Este comportamento está diretamente relacionado ao incremento da frequência gestual quando do incremento da velocidade de nado, como uma estratégia adotada por nadadores em situação de incremento agudo da velocidade de nado, como demonstrado anteriormente por Castro et al. (2005) para o nado crawl em nadadores competitivos e triatletas, e por Mazzola et al. (2008) também no nado crawl, mas entre nadadores recreacionais.

As durações relativas das fases da braçada apresentaram modificações com o incremento da velocidade de nado (entre V200 e Vmáx). Houve uma maior duração das fases propulsivas da braçada, devido ao maior tempo relativo das fases $\mathrm{B}$ e $\mathrm{C}$ e à diminuição na duração relativa da fase $\mathrm{A}$, ao passo que a velocidade de nado aumentou (Tabela 2). Estes resultados vão ao encontro do comportamento descrito na literatura, sobre a adaptação na duração das fases da braçada nas quatro técnicas de nado (CHOLLET et al., 2000; SEIFERT et al., 2004; SEIFERT; CHOLLET, 2005; CHOLLET et al., 2006; CHOLLET et al., 2008; SCHNITZLER et al., 2008). De modo específico, no nado borboleta, entre as velocidades relativas aos $400 \mathrm{~m}$ e 50 m, Chollet et al. (2006), encontraram incremento de 4\% na fase B (de 20 a 24\% da duração média do ciclo) e I, $1 \%$ na fase $C$ (de 21 a 22, $1 \%$ da duração média do ciclo), enquanto que a fase A reduziu em 10\% (de 36, I a 26,3\% da duração média do ciclo). Mas esses resultados (CHOLLET et al., 2006) foram obtidos após análise de 14 nadadores de elite, com média de melhor tempo na prova de $100 \mathrm{~m}$ borboleta, de $57 \mathrm{~s}$, e entre V400 e V50, diferentemente do presente estudo piloto, que contou com quatro nadadores de nível competitivo provavelmente inferior.

Já em relação aos tempos relativos de execução das pernadas (Tabela 3), pode-se verificar incremento da duração relativa das fases descendentes (propulsivas) e redução da duração relativa das fases ascendentes (não propulsivas), ao passo que a velocidade de nado aumentou. Este comportamento foi parcialmente similar ao encontrado por Chollet et al. (2006), que encontraram incremento de aproximadamente $2,4 \%$ no tempo relativo de duração da primeira descendente (de 15, I para 17,5\%, entre velocidade relativa aos 400 m e velocidade relativa aos 50 m), e redução de aproximadamente 3,5\% na duração relativa da primeira ascendente (entre 33,4 para 29,9\%, entre velocidade relativa aos $400 \mathrm{~m}$ e velocidade relativa aos 50 m). As outras fases não apresentaram, para Chollet et al. (2006), comportamento consistente de incremento ou redução de suas durações relativas.

O comportamento de TTG, com o incremento da VN, indica um maior grau de sincronismo entre os pontos-chave da braçada e da pernada nas velocidades de nado mais elevadas. No presente estudo, esse comportamento se deve principalmente à redução nos tempos relativos T1, T2 e T4 (Tabela 4), ao contrário 
dos achados de Chollet et al. (2006) e Seifert et al. (2008), os quais verificaram adaptações somente nos valores de TI e T2 em nadadores de elite. Essas diferenças, nas comparações entre os estudos, podem estar relacionadas à diferença de nível competitivo dos nadadores participantes de ambos os estudos.

TI : entre V200 e V50, os nadadores apresentaram um intervalo de tempo entre o ponto de entrada das mãos na água e o início da fase descendente da primeira pernada, o que pressupõe um período em deslize, sem geração de propulsão, já que não há movimentos propulsivos. Com o incremento da $\mathrm{VN}$, houve uma redução nesse tempo relativo, como mostrado por Chollet et al. (2006), Seifert et al. (2007) e Seifert et al. (2008). Em Vmáx, os nadadores acabaram precipitando o início da ação propulsiva da pernada, iniciando-a antes da entrada das mãos na água $(T I<0 \%)$. Isso ocasionou uma perda de sincronismo entre as ações de braços e pernas. Agudamente (quando há a necessidade de incremento da velocidade, sem considerar efeitos do treinamento a longo prazo), incrementos na $\mathrm{VN}$ são obtidos por incrementos na frequência de ciclos de braçadas (CASTRO et al., 2005), o que pode ter dificultado a manutenção de uma coordenação adequada em Vmáx, que foi uma velocidade superior às VN relativas aos eventos competitivos de $200 \mathrm{~m}, 100 \mathrm{~m}$ e $50 \mathrm{~m}$.

T2: os nadadores apresentaram um tempo sem que ocorressem ações propulsivas entre o final da ação propulsiva da primeira pernada e o início da fase de puxada. Essa diferença de tempo entre os pontos-chave da pernada e da braçada diminuiu entre V200 e Vmáx, indo ao encontro dos achados de Chollet et al. (2006), Seifert et al. (2007) e Seifert et al. (2008). Esse comportamento está relacionado à diminuição na duração relativa da fase de entrada e apoio, observada com o incremento da VN (CHOLLET et al., 2006).

T4: entre V200 e Vmáx, os nadadores apresentaram valores negativos de $\mathrm{T} 4$, ou seja, houve um intervalo de tempo entre o final da fase descendente da segunda pernada e o final da fase de empurrada. Com o incremento da velocidade de nado, ocorreu uma diminuição nesse intervalo de tempo, levando a um maior sincronismo entre as ações da braçada e da pernada.

Estes resultados vão de encontro aos apresentados por Chollet et al. (2006) e Seifert et al. (2008), os quais não observaram adaptações nos valores de T4. Possivelmente essa divergência se deva à diferença entre o nível de desempenho dos sujeitos avaliados no presente estudo, e o dos sujeitos avaliados nos estudos prévios (SEIFERT et al., 2007). Os participantes deste estudo são atletas de nível regional, os quais demonstraram dificuldades no controle da técnica de borboleta nas velocidades de nado mais baixas, o que pode ter influenciado nos valores de T4, ao contrário dos participantes dos estudos prévios, os quais apresentavam um nível de desempenho mais elevado. 
Em termos absolutos (Tabela I), os resultados de duração média dos ciclos de braçada e pernada foram similares entre ciclos inspiratórios e ciclos não-inspiratórios, com tendência a redução do tempo para os ciclos não-inspiratórios. Com relação ao efeito das ações inspiratórias, houve uma menor duração relativa da fase A (Tabela 2) e um consequente incremento na duração propulsiva da braçada, quando realizados ciclos de braçada não-inspiratórios. Essa redução na duração da fase $\mathrm{A}$ possivelmente está relacionada a uma maior velocidade de deslocamento horizontal das mãos, como observado por Barbosa (2000), o qual comparou as técnicas de borboleta com ciclos de braçada não-inspiratórios e ciclos de inspiração frontal.

Com a execução de ciclos de inspiração frontal, há uma maior amplitude de deslocamento vertical (BARBOSA et al., 1999; BARBOSA, 2000), o que em parte pode explicar a maior duração da fase de entrada e apoio. Barbosa (2000) encontrou, para inspiração frontal, média de 0,480 \pm 0,058 m para o deslocamento vertical, em comparação à média de 0,204 \pm 0,031 m para ciclos não-inspiratórios, ao comparar diferentes técnicas de nado borboleta em nadadores de distintos níveis competitivos. Outra questão a ser considerada é que, em condição de ciclos não-inspiratórios, o nadador realiza uma flexão cervical, a qual facilita o final da fase de recuperação da braçada (BARBOSA, 2004), proporcionando uma maior velocidade de deslocamento das mãos durante a fase de entrada e apoio (BARBOSA, 2000; BARBOSA et al., 2003).

A execução de ciclos de inspiração frontal acarretou um menor sincronismo entre os pontos-chave de braçada e de pernada (Tabela 4), representado pela maior duração dos tempos relativos TI (3,9 \pm 0,8\% vs. 2,2 \pm 1 , I \%) e T2 (-20,8 \pm 2,4\% vs. - 19,0 $\pm 2,3 \%)$. Esse comportamento está diretamente relacionado à duração da fase A, já que TI e T2 ocorrem justamente entre o ponto de entrada das mãos na água ( $\mathrm{TI}$ ) e o ponto imediatamente anterior ao movimento das mãos para trás (T2).

\section{CONCLUSÃO}

Tanto o incremento da velocidade de nado, quanto a execução de ciclos de inspiração frontal, provocaram adaptações na coordenação do nado borboleta. Essas adaptações são referentes a maior sincronismo entre ações de braços e pernas, bem como maior duração das fases propulsivas, nas velocidades mais elevadas (velocidade máxima e relativa aos $50 \mathrm{~m}$ ) e quando executados ciclos de braçada não-inspiratórios. 
Quando o nado borboleta é executado em baixas velocidades de nado, ocorre uma maior descontinuidade, devido ao maior tempo em que não há ações propulsivas, pressupondo maior tempo em deslize dos nadadores. Ainda, quando orientados a nadar em velocidades de nado extremas, os nadadores devem focar na melhor continuidade das ações propulsivas. Assim, parece ser adequado o treinamento do nado borboleta em ritmo de prova, já que a execução repetitiva de um gesto esportivo de maneira adequada pode levar a incrementos de desempenho.

A análise individual dos efeitos de ciclos de inspiração frontal sobre a coordenação de nado torna-se importante para desenvolver estratégias adequadas para a frequência de ações inspiratórias durante eventos competitivos.

\section{Butterfly stroke coordination: a pilot study about effects on speed and on inspiratory actions}

ABSTRACT: The purpose of this pilot study was to describe the effects of pace and breathing actions on the coordinative parameters of the butterfly stroke. Four regional level swimmers participated of this study (mean performance level: $69 \pm 3.6 \%$ of the world record in the 100-m butterfly). Increasing speed, similar results were observed for both frontal breathing and non-breathing conditions. From the $200 \mathrm{~m}$ pace to the maximal speed trial, there is a decrease in the absolute duration of arm stroke (from $2.01 \pm 0.43 \mathrm{~s}$ to $1.07 \pm 0,09 \mathrm{~s}$ ), increasing the relative duration of propulsive phases (pull phase: from $14.6 \pm 3.2 \%$ to $22.8 \pm 4.7 \%$; push phase: from 12.9 \pm 1.0 to $19.1 \pm 0.9 \%)$. Regarding to the inter-limb coordination, there is a higher continuity between the arm and leg stroke key points.

KEY-WORDS: Swimming; video analysis; butterfly stroke; coordination.

Coordinación del estilo mariposa: estudio previo sobre los efectos de la velocidad de nado y de las acciones inspiratorias

RESUMEN: La propuesta de este estudio piloto fue verificar los efectos de la velocidad de nado y de los ciclos inspiratorios sobre los parámetros coordinativos del nado mariposa. Participaron cuatro nadadores de nivel regional, con desempeño medio de $69 \pm 3,6 \%$ del record mundial en la prueba de $100 \mathrm{~m}$ mariposa. En respuesta al aumento de la velocidad de nado, entre los principales resultados, hubo: reducción en la duración absoluta de los ciclos (de 2,01 \pm 0,43 s para 1,07 $\pm 0,09$ s, entre la velocidad relativa a los 200 m e velocidad máxima en $25 \mathrm{~m}$, con inspiración frontal y similar comportamiento para la condición sin respirar); un marcado aumento en la duración de las fases propulsivas de la brazada (fase B de 14,6 \pm 3,2\% para 22,8 \pm 4,7\% y fase C de 12,9 1 1,0 para 19,1 \pm 0,9\% con inspiración frontal y similar comportamiento para la condición sin respirar).

PALABRAS CLAVE: Natación; análisis de vídeo; nado mariposa; coordinación. 


\section{REFERÊNCIAS}

ALVES, F., CUNHA, P., GOMES-PEREIRA, J. Kinematic changes with inspiratory actions in butterfly swimming. In: KESKINEN, K. L.; KOMI, P. V.; HOLLANDER, A. P. Biomechanics and Medicine in Swimming VIII. Jyvaskyla: Gummerus, 1999. p. 9-I4.

BARBOSA, T. Análise tridimensional da cinemática da técnica de Mariposa ao realizarem-se ciclos de inspiração frontal, ciclos de inspiração lateral e ciclos não inspiratórios. 2000. I60 f. Dissertação (Mestrado em Desporto para Crianças e Jovens) - Faculdade de Ciências do Desporto e de Educação Física, Universidade do Porto, Porto, 2000.

Os mitos e as realidades sobre a inspiração lateral na técnica de mariposa, 2002. Federação Portuguesa de Natação, Portugal. Disponível em: < http://www.fpnatacao.pt/ formacao_pedagogica_detalhe.php?idArtigo=498\&idCategoria=39>. Acesso em: 20 ago. 2009.

Caracterização biofísica da técnica de mariposa). Bragança: Instituto Politécnico de Bragança, 2004.

BARBOSA, T. et al. Kinematical modifications induced by the introduction of the lateral inspiration in butterfly stroke. In: KESKINEN, K. L.; KOMI, P. V.; HOLLANDER, A. P. Biomechanics and Medicine in Swimming VIII. Jyvaskyla: Gummerus, 1999. p. I5-20.

BARBOSA, T. M. et al. Characterization of hand's velocity in butterfly stroke according to the breathing technique. In: ANNUAL CONGRESS OF THE EUROPEAN COLLEGE OF SPORTS SCIENCES, 8., 2003, Salzburg. Annals.... Salzburg: European College of Sports Sciences, 2003.

CASTRO, F. A. S. et al. Cinemática do nado "crawl" sob diferentes intensidades e condições de respiração de nadadores e triatletas. Revista Brasileira de Educação Física e Esportes, São Paulo, v. 19, n. 3, p. 223-232, jul/set. 2005.

CHOLLET, D.; CHALIES, S.; CHATARD, J. C. A new index of coordination for the crawl: description and usefulness. International Journal of Sports Medicine, Stuttgart, v. 2 I, n. I, p. 54-59, jan. 2000.

CHOLLET, D. et al. A new index of coordination for the crawl: description and usefulness. International Journal of Sports Medicine, Stuttgart, v. 2 I , n. I, p. 54-59, jan. 2000.

Evaluation of arm-leg coordination in flat breaststroke. International Journal of Sports Medicine, Stuttgart, v. 25, n. 7, p. 486-495, out. 2004.

Arm to leg coordination in elite butterfly swimmers. International Journal of Sports Medicine, Stuttgart, v. 27, n. 4, p. 322-329, abr. 2006.

Arm coordination in elite backstroke swimmers. Journal of Sports Sciences, London, v. 26, n. 7, p.675-682, maio 2008. 
MAZZOLA, P. et al. Cinemática do nado crawl de nadadores recreacionais. Revista Brasileira de Ciência e Movimento. v. I6, n. 3, 2008.

SCHNITZLER, C. et al. Arm coordination adaptations assessment in swimming. International Journal of Sports Medicine, Suttgart, v. 29, n. 6, p. 480-486, june 2008.

SEIFERT, L. et al. Effect of swimming velocity on arm coordination in the front crawl: a dynamic analysis. Journal of Sports Sciences, London, v. 22, n. 7, p. 65I-660, july 2004.

Effect of expertise on butterfly stroke coordination. Journal of Sports Sciences,

London, v. 25, n. 2, p. 131 - | 4 |, jan. 2007.

Differences in spatial-temporal parameters and arm-leg coordination in butterfly stroke as a function of race pace, skill and gender. Human Movement Science, Amsterdam, v. 27, n. I, p. 96- I I I, feb. 2008.

SEIFERT, L.; CHOLLET, D. A new index of flat breaststroke propulsion: a comparison of elite men and women. Journal of Sports Sciences, London, v. 23, n. 3, p. 309-320, mar. 2005.

Modelling spatial-temporal and coordinative parameters in swimming. Journal of Science and Medicine in Sport, Belconnen, v. I2, n. 4, p. 495-499, july 2009.

Recebido: 21 out. 2009

Aprovado: 24 ago. 2011

Endereço para correspondência:

Ricardo Peterson Silveira

LAPEX - ESEF - UFRGS

Rua Felizardo, 750

Bairro Jardim Botânico

Porto Alegre - RS

90690-200 